\title{
Hydrophobic and oleophilic cotton fabrics for efficient oil-water separation through low-pressure plasma polymerization
}

\author{
L Ghorbani ${ }^{1}$, A Khatibi ${ }^{2}$, and B Shokri ${ }^{1,2}$ \\ 1. Laser and Plasma Research Institute, Shahid Beheshti University, Tehran, Iran \\ 2. Faculty of Physics, Shahid Beheshti University, Tehran, Iran
}

(Received 23 May 2018; in final form 04 May 2019)

\begin{abstract}
In the recent years, the increase of industrial effluents in the petrochemical sector, particularly the leakage of oil and the draining of industrial effluents in rivers, has created serious environmental hazards and a huge economic loss in the world. In the past decade, the use of hydrophobic and oleophilic fabrics has been considered as a way to clean up contaminants through the absorption and separation of pollutants from industrial effluents. In this research, the lowpressure plasma polymerization method based on eco-friendly materials like Polydimethylsiloxane was used to fabricate hydrophobic and oleophilic cotton fabric. Also, a low-pressure oxygen plasma pre-treatment was performed before plasma polymerization to improve the bonding between the created layer and cotton fabric. Contact angle test and absorption capacity test were then used to represent the hydrophobicity of the coated fabric and to measure the absorbance ability of different oils. Also, scanning electron microscopy (SEM) was used to observe morphological changes on the surface of cotton fibers and Infrared Fourier transform (FTIR-ATR) spectroscopy was applied to detect the chemical bonds created on the surface of fibers. Water-oil separation efficiency test and laundering test were conducted to determine the separation rate and to represent the durability of the coated cotton, respectively. The water contact angle of the coated cotton fabric was $143 \pm 3$ and this high hydrophobicity behavior almost remained after the 10 cycle laundering. Also, SEM results showed that the surface of fibers was covered by a random distribution of several microscale structures or a hierarchical surface structure like the lotus leaf. Our Water-oil separation tests further demonstrated that coated fabrics had a separation efficiency between 80 until 100 percent for most of the industrial oil, even after 15 cycles at $250^{\circ} \mathrm{C}$ and $900^{\circ} \mathrm{C}$. These results, therefore, indicate that the coated cotton fabrics with plasma polymerization method could have a high potential for application in water-oil separation and selective oil absorption. The fabrics are promising for the development of an environmentally friendly and recyclable separation method of oil from water. These fabrics are considered as a promising method for separating oil from water with biocompatibility and reusability.
\end{abstract}

Keywords: polydimethylsiloxane, plasma polymerization, hydrophobic-oleophilic fabrics, water-oil separation

For full article, refer to the Persian section 